\title{
Resistant type 2 amiodarone-induced thyrotoxicosis responsive to cholestyramine as an adjunctive therapy
}

\author{
Authors: Aisha Rummaan, ${ }^{\mathrm{A}}$ Maryam Maryam, ${ }^{\mathrm{B}}$ Awais Ali, ${ }^{\mathrm{A}}$ Snigdhendu Mandal ${ }^{\mathrm{C}}$ and Tamar Saeed ${ }^{\mathrm{D}}$
}

\begin{abstract}
Amiodarone is a class 3 antiarrhythmic drug which may be associated with thyroid dysfunction. Amiodarone-induced thyrotoxicosis (AIT) is classified as type 1 (AIT 1; which may develop in the presence of latent autoimmune hyperthyroid condition) or type 2 (AIT 2; which develops in an apparently normal thyroid resulting from destructive thyroiditis). AIT 1 routinely requires treatment with thionamides, whereas AIT 2 is treated with steroids. Resistance to the conventional treatment of hyperthyroidism is not commonly found in clinical practice. This report discusses a case of AIT 2 resistant to conventional treatment. Despite being on high doses of carbimazole and steroids (prednisolone), the patient remained thyrotoxic. Cholestyramine, a bile salt sequestrant, was used as an adjunctive therapy resulting in significant clinical and biochemical improvement. The patient subsequently became euthyroid and is being followed up in endocrine clinic.
\end{abstract}

KEYWORDS: amiodarone-induced thyrotoxicosis, AIT, cholestyramine, resistant thyrotoxicosis

DOI: $10.7861 /$ clinmed.2021-0482

\section{Case presentation}

A 49-year-old man with background history of atrial fibrillation, hypertension and irritable bowel syndrome presented to ambulatory emergency care (AEC) with complaints of tiredness, sweating, diarrhoea, palpitations and weight loss of 4.5 stone over 5 months. He previously had unsuccessful direct current cardioversion. His regular medications included amiodarone, bisoprolol and rivaroxaban. He was not known to have previous thyroid illnesses. There was no family history of thyroid disorders. He was an ex-smoker with 5 pack-years smoking history and there

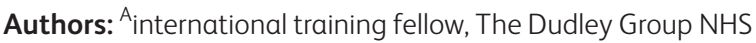
Foundation Trust, Dudley, UK; Bjunior clinical fellow, The Dudley Group NHS Foundation Trust, Dudley, UK; ' ${ }^{\text {consultant acute }}$ physician, The Dudley Group NHS Foundation Trust, Dudley, UK; ${ }^{D}$ consultant endocrinologist, The Dudley Group NHS Foundation Trust, Dudley, UK was no history of alcohol consumption or substance abuse. He was a ground maintenance worker.

On examination, the patient was tachycardiac with heart rate of 95 beats per minute and had a normal blood pressure of 122/68 $\mathrm{mmHg}$. He had obvious tremors of his hands but rest of his clinical examination was unremarkable.

His thyroid function tests at presentation showed free thyroxine (T4) of more than $100 \mathrm{pmol} / \mathrm{L}$ (normal range 12.0-22.10), free triiodothyronine (T3) of $36 \mathrm{pmol} / \mathrm{L}$ (normal range 3.1-6.8) and suppressed thyroid stimulating hormone (TSH).

\section{Initial management and prognosis}

In view of presentation and investigations, the diagnosis of amiodarone-induced thyrotoxicosis (AIT) was made. Due to the ambiguity about the type of AIT, he was commenced on carbimazole $30 \mathrm{mg}$ once daily and prednisolone $20 \mathrm{mg}$ daily. The dose of bisoprolol was increased to $10 \mathrm{mg}$ daily. His thyroid peroxidase (TPO) antibodies and TSH receptor antibodies (TRAB) came back negative. After 3 weeks of treatment, his thyroid functions remained almost the same. He had Tc-99m pertechnetate scintigraphy, which showed reduced uptake (Fig 1a), and an ultrasound of the thyroid, which showed normal vascularity (Fig 1b). Investigations led to the diagnosis of type 2 AIT. The patient was reviewed after 3 weeks, however, there was no clinical or biochemical improvement. When cholestyramine was added to his treatment, which is a known bile acid sequestrant, the patient showed dramatic improvement of his symptoms after a week. Biochemical improvement was evident in the form of decline in free T4 (63.4 pmol/L) and free T3 (12.1 pmol/L). The dose of prednisolone was subsequently increased to $40 \mathrm{mg}$ daily and carbimazole was slowly withdrawn. The free T4 and T3 continued to improve till they normalised, then all the treatment for the thyroid stopped. The rest of his blood tests remained normal throughout.

\section{Case progression and outcome}

The patient was followed-up in the endocrine clinic. After 21 weeks of treatment, prednisolone was weaned off. When the patient became euthyroid clinically as well as biochemically then cholestyramine was stopped. His latest thyroid functions showed free T4 of $16.9 \mathrm{pmol} / \mathrm{L}$, free T3 of $5.5 \mathrm{pmol} / \mathrm{L}$ and TSH of 2.06 $\mathrm{mIU} / \mathrm{L}$. He regained the weight that he lost initially and is also being reviewed in cardiology clinic for atrial fibrillation. 

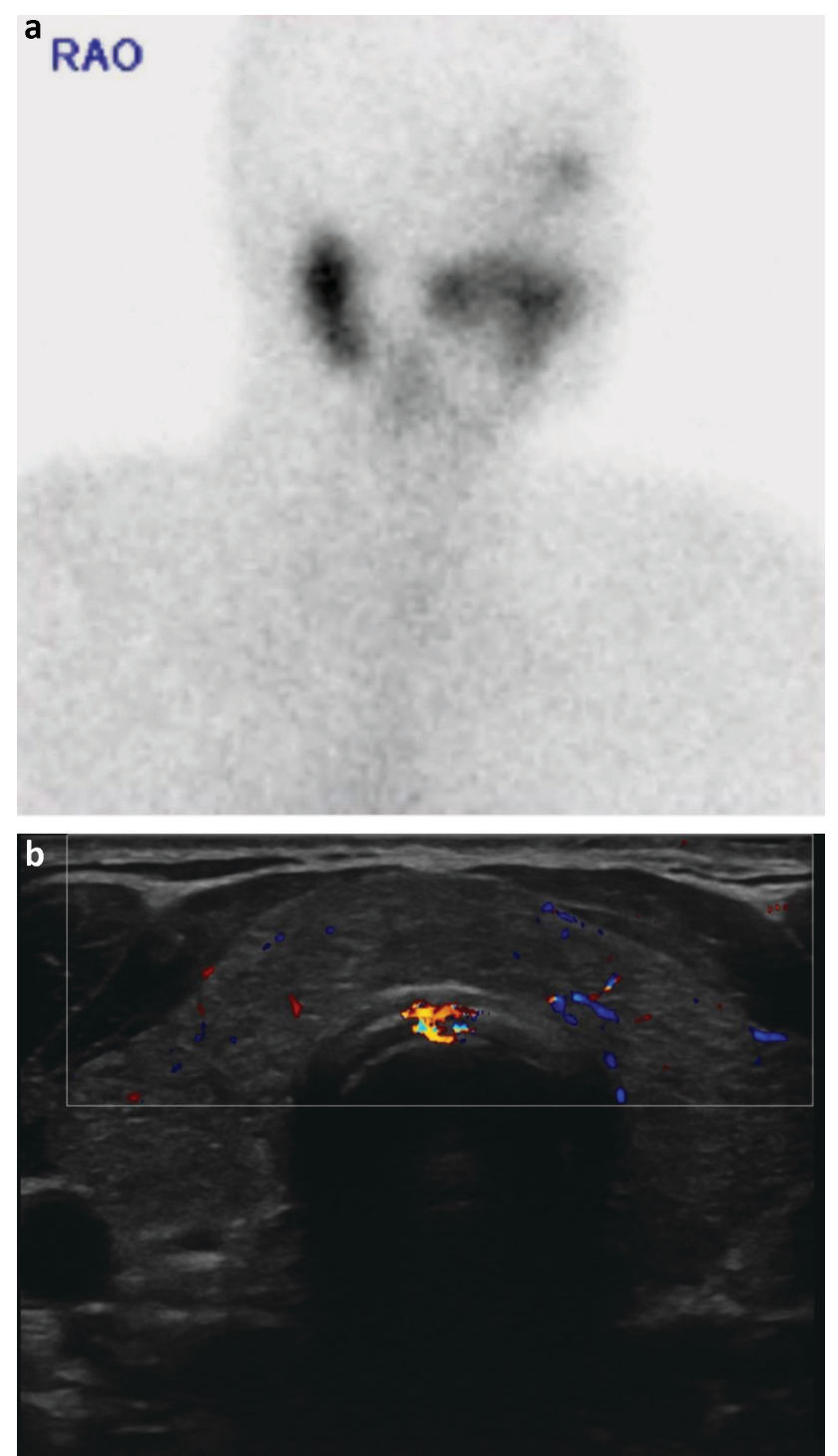

Fig 1. Thyroid imaging. a) Tc-99m pertechnetate scintigraphy showing reduced uptake. b) Ultrasound showing normal vascularity.

\section{Discussion}

Prolonged treatment with amiodarone can affect thyroid function. Amiodarone is structurally similar to T4 and contains a high iodine content. Elimination half-life of amiodarone is on average 58 days. ${ }^{1}$ However, abrupt cessation of amiodarone will not have a significant effect. Therefore, continuing amiodarone is often difficult to decide, hence cardiology input is required. ${ }^{2}$

Thyroid hormones are metabolised mainly in the liver by conjugation to glucuronides through which it enters enterohepatic circulation. ${ }^{2}$ Cholestyramine is an ion exchange resin which interferes with endogenous thyroid hormone absorption seen in hyperthyroid patients in previous studies. ${ }^{3}$

A randomised controlled trial showed rapid decline of thyroid hormones in hyperthyroid patients who received cholestyramine in addition to methimazole and propranolol in comparison with conventional therapy alone (ie just methimazole and propranolol). ${ }^{3}$
A case report of refractory Graves' hyperthyroidism also shows a response to cholestyramine. ${ }^{4}$

Another case report of a huge multi-nodular goitre with obstructive symptoms, refractory to 3-weeks' treatment of conventional therapy (including dexamethasone, carbimazole and propranolol) shows a rapid decrease of free T4 by more than $30 \%$ only 5 days after introducing cholestyramine, in addition to ongoing treatment and normalisation of free T4 7-12 days after initiation of cholestyramine. ${ }^{5}$

The 2018 European Thyroid Association recommends oral glucocorticoids as the first-line treatment for AIT 2 with moderateto-severe thyrotoxicosis. ${ }^{6}$

\section{Conclusion}

We report a case of refractory AIT 2 that failed to respond to conventional treatment. However, adding cholestyramine as an adjunctive therapy showed significant clinical and biochemical improvement; we suggest this could be considered as a treatment option in resistant cases of thyrotoxicosis.

\section{Key points}

> Cholestyramine may be helpful in refractory cases of thyrotoxicosis and can also be used in AIT 2 before proceeding to salvage thyroidectomy, if needed.

> Amiodarone has a long elimination half-life, so abrupt cessation of amiodarone may not be helpful.

> Thyroid technetium uptake scintigraphy has no role in making the initial diagnosis in regard to the type of AIT due to high iodine content of amiodarone and its long half-life.

> Ultrasound of the thyroid may be useful in differentiating the type of AIT, initially. However, in our case, it was not significant as was done late in the course.

\section{References}

1 Rajeswaran C, Shelton RJ, Gilbey SG. Management of amiodaroneinduced thyrotoxicosis. Swiss Med Wkly 2003;133:579-85.

2 Tsai WC, Pei D, Wang TF et al. The effect of combination therapy with propylthiouracil and cholestyramine in the treatment of Graves' hyperthyroidism. Clin Endocrinol (Oxf) 2005;62:521-4.

3 Mercado M, Mendoza-Zubieta V, Bautista-Osorio R, Espinoza-de los Monteros AL. Treatment of hyperthyroidism with a combination of methimazole and cholestyramine. J Clin Endocrinol Metab 1996;81:3191-3.

4 Sebastián-Ochoa A, Quesada-Charneco M, Fernández-García D et al. Dramatic response to cholestyramine in a patient with Graves' disease resistant to conventional therapy. Thyroid 2008;18:1115-17.

5 Alswat KA. Role of cholestyramine in refractory hyperthyroidism. Am J Case Rep 2015;16:486-90.

6 Bartalena L, Bogazzi F, Chiovato L et al. 2018 European Thyroid Association (ETA) Guidelines for the Management of AmiodaroneAssociated Thyroid Dysfunction. Eur Thyroid J 2018;7:55-66.

7 Cardenas GA, Gustavo AC, Cabral JM et al. Amiodarone-induced thyrotoxicosis: Diagnostic and therapeutic strategies. Cleve Clinic J Med 2003;70:624-31

Address for correspondence: Dr Tamar Saeed, Diabetes Centre, North Block, Russells Hall Hospital, The Dudley Group NHS Foundation Trust, Pensnett Road, Dudley DY1 2HQ, UK. Email: tamar.saeed@nhs.net Twitter: @SaeedTamar 